\title{
Dynamiques de création et trajectoires d'Ateliers de transformation collectifs (ATC) dans la région Occitanie
}

Dynamics of creation and trajectories of collective transformation workshops in the Occitanie region

\section{Agnès Terrieux, Mohamed Gafsi et Ugo Fiaschi}

\section{OpenEdition \\ Journals}

Édition électronique

URL : https://journals.openedition.org/developpementdurable/18804

DOI : 10.4000/developpementdurable.18804

ISSN : 1772-9971

Éditeur

Association DD\&T

\section{Référence électronique}

Agnès Terrieux, Mohamed Gafsi et Ugo Fiaschi, « Dynamiques de création et trajectoires d'Ateliers de transformation collectifs (ATC) dans la région Occitanie », Développement durable et territoires [En ligne], Vol. 12, $n^{\circ} 1$ | Mai 2021, mis en ligne le 26 mai 2021, consulté le 31 mai 2021. URL : http:// journals.openedition.org/developpementdurable/18804; DOI : https://doi.org/10.4000/ developpementdurable.18804

Ce document a été généré automatiquement le 31 mai 2021.

\section{(c) (†) (8)}

Développement Durable et Territoires est mis à disposition selon les termes de la licence Creative Commons Attribution - Pas d'Utilisation Commerciale 4.0 International. 


\title{
Dynamiques de création et trajectoires d'Ateliers de transformation collectifs (ATC) dans la région Occitanie
}

\author{
Dynamics of creation and trajectories of collective transformation workshops in \\ the Occitanie region
}

Agnès Terrieux, Mohamed Gafsi et Ugo Fiaschi

Dans un contexte d'interrogation du modèle de développement issu de la modernisation, des agriculteurs ont développé des formes alternatives d'exploitation dans lesquelles la transformation des produits et leur vente en circuits courts jouent un rôle important (Muller et al., 1989; Van der Ploeg et al., 2009; Mondy, 2010 ; Poisson et Saleilles, 2012 ; Gafsi, 2017). Dans ce contexte de transition agricole, divers projets de création de structures collectives ont émergé, sous le nom d'ateliers de transformation collectifs (ATC) (Mondy et Terrieux, 2010; Poisson et Saleilles, 2012). Un ATC est un atelier agroalimentaire géré par un collectif d'agriculteurs-utilisateurs maitrisant le processus de transformation. La commercialisation est propre à chacun; mais dans certains cas une partie des produits peut être commercialisée collectivement. Des ateliers types sont identifiables en fonction des produits transformés (viandes multiespèces, volailles, fruits et légumes, céréales, miel, etc.). Ces ATC représentent des configurations d'action collective de proximité (Lucas et al., 2014) mobilisant différentes formes de coopération agricole. Ils sont apparus en France depuis une trentaine d'années. Ces nouvelles structures sont relativement récentes, comparées au mouvement coopératif agricole qui a débuté à la fin du XIX siècle. Elles se distinguent aussi de la coopération traditionnelle, par leur logique d'émancipation par rapport au modèle agricole productiviste (Mondy, 2015). Elles s'inscrivent dans les modèles agroalimentaires alternatifs (Goodman, 2004 ; Maye, 2013).

2 Si les travaux sur la coopération agricole ont été nombreux (Nicolas, 1988; Koulytchizky et Mauget, 2003; Filippi et al., 2008), les recherches portées 
spécifiquement à l'émergence de nouvelles structures collectives restent cependant rares. En dehors de quelques travaux fondateurs (Vincq et al., 2010; Mundler et Valorge, 2015), peu de recherches ont été consacrées à l'analyse de l'émergence et de l'évolution de ces nouvelles structures collectives. Fondées sur des principes de solidarité entre adhérents et des démarches participatives, elles semblent a priori pouvoir être épargnées par la distanciation des adhérents décrite dans les structures coopératives traditionnelles (Barraud-Didier et al., 2012). Mais certains ATC rencontrent d'autres types de difficultés, en raison de leur incapacité à renouveler leurs adhérents, ou plus simplement parce que leur filière est en crise (aviculture par exemple) ; ce qui conduit à poser la question des conditions de leur pérennité.

Partant d'enquêtes réalisées en 2019, le présent article vise deux objectifs : comprendre les processus qui ont amené à la création des ATC et apporter un éclairage sur leurs trajectoires et les facteurs de leur pérennité. Il s'appuie pour cela sur l'analyse rétrospective de l'évolution de 18 ATC dans le sud-ouest de la France.

4 Dans une première section, nous présenterons notre cadre analytique et méthodologique. La deuxième section exposera les résultats des analyses. Elle montrera dans un premier temps un portrait des différents ATC étudiés et les processus de leur mise en place. Puis seront présentées les trajectoires types des ATC, lors des dix dernières années, ainsi que les facteurs de pérennité. La troisième section discutera les résultats d'analyse et conclura sur les enseignements à tirer de ces expériences sur les conditions de l'action collective.

\section{Cadre analytique et méthodologique}

\subsection{Cadre d'analyse : action collective et pérennité organisationnelle}

5 La thématique de l'action collective a fait l'objet de nombreux travaux scientifiques au cours de ces dernières années, couvrant plusieurs champs disciplinaires (Amblard et al., 2018). Les travaux en économie s'intéressent aux choix des agents et aux mécanismes de coordination qui conduisent à l'émergence de l'action collective et à sa réussite (Olson, 1965 ; Ostrom, 1990 ; Meinzen-Dick et al., 2004). La sociologie a mis en avant l'importance des relations de pouvoir entre acteurs et l'établissement des règles (Crozier et Friedberg, 1977; Friedberg, 1993). Les sciences de gestion considèrent l'action collective comme une construction conjointe de savoirs et de relations (Hatchuel et Weil, 1992). Cela englobe des dimensions de mise en commun de moyens, de coopération et de conflits, d'apprentissage entre acteurs, et de réalisations communes mesurables (Hatchuel, 2000).

6 La littérature montre que la plupart des définitions de l'action collective mettent l'accent sur ses quatre dimensions fondamentales (Ostrom, 1990; Hatchuel, 2000 ; Meinzen-Dick et al., 2004) :

1. les acteurs : l'implication d'un groupe d'acteurs autonomes, de diverses catégories (individus, institutions, associations, etc.), portant des enjeux et intérêts plus ou moins divergents et ayant des degrés variés d'influence et d'implication ;

2. les objectifs: le partage d'un ou plusieurs objectifs au sein du groupe d'acteurs, ce qui fonde l'intérêt pour l'action collective, en lien par exemple avec la gestion de ressources communes ; 
3. la gouvernance : l'établissement de dispositifs (règles, normes, conventions, etc.) pour assurer une meilleure coordination des parties prenantes pour la prise de décision et la conduite de l'action (Ostrom, 1990). Ces dispositifs de gouvernance sont souvent d'ordre général, permettant de cadrer l'action et laissant place à des ajustements et des arrangements au cours du temps. Ils se traduisent, dans le cadre d'une structure collective, par l'établissement d'un règlement intérieur, de règles de bonnes pratiques, des modalités de prise de décision, etc. ;

4. la mesure de résultats: l'existence de procédure et d'outils de suivi et d'évaluation des résultats de l'action (Girin, 1990), permettant d'apprécier ses performances et d'en partager les bénéfices; ce qui constitue un élément fondamental pour la continuité de l'action collective.

7 Ces quatre dimensions sont indispensables à toute action collective. Cependant, le processus de sa mise en place nécessite la prise en compte de trois éléments. Premièrement, ce processus peut prendre, dans un premier temps du moins, la forme d'actions diffuses et informelles, spontanées et émergentes, qui se structurent et se formalisent par la suite, progressivement et chemin faisant. Ce processus ne constitue pas toujours une action délibérée, formelle, résultant d'un choix rationnel d'acteurs concernant un enjeu ou une ressource commune. Deuxièmement, dans le domaine agricole et de la gestion des ressources naturelles, les processus d'action multi-acteurs sont souvent marqués par les conditions territoriales, en lien avec les dimensions matérielles (caractéristiques des ressources considérées), organisationnelles et/ou idéelles des territoires (Amblard et al., 2018). Troisièmement, cette dimension locale et territoriale peut être saisie par la notion de proximité (Lucas et al., 2014). Toutefois, ces actions n'échappent pas à l'influence des évolutions socio-économiques globales ou des politiques publiques (Amblard et al., 2018). C'est le cas par exemple des démarches d'action collective visant à accompagner les stratégies de diversification des agriculteurs (Muller et al., 1989 ; Van de Ploeg et al., 2009 ; Gafsi, 2017).

8 Une condition essentielle de la pérennité d'une ATC se trouve dans sa capacité à répondre autant à des évolutions internes à la structure qu'aux impacts d'événements extérieurs. Comment, dans la durée, faire vivre le projet d'ATC, qui est au cœur de l'action collective et affronter les bouleversements? La notion de pérennité peut renvoyer dans un sens commun à la capacité de continuer dans le temps (Godelier, 2009). Des structures pérennes sont des entités qui ont perduré et qui continuent à exister. Mais cette vision survalorise la continuité ou la stabilité au détriment du changement ; or, pour se maintenir, il faut évoluer, être en phase avec les évolutions du contexte. La notion de pérennité organisationnelle (Mignon, 2009) traduit bien cette injonction contradictoire. Selon l'auteur, il s'agit de "la capacité pour une entreprise d'initier ou de faire face au cours de son histoire à des bouleversements externes ou internes tout en préservant l'essentiel de son identité » (p. 75). L'entreprise, en tant qu'organisation, doit évoluer tout en gardant ses fondements. La pérennité d'une organisation comporte alors deux dimensions contradictoires : changement et continuité. C'est la combinaison récursive et non séquentielle de ces deux dimensions qui conduit à la pérennité organisationnelle.

9 La dimension, relative au changement, renvoie aux capacités et aux processus d'adaptation permettant à une organisation de s'ajuster aux évolutions d'origine externe ou interne. Les évolutions externes, pour les ATC, concernent l'évolution du contexte agricole en général (nouvelles attentes sociales en matière de qualité de produits et de préservation de l'environnement, par exemple), ou d'une filière en 
particulier (crise récente de la filière avicole, par exemple). Pour les évolutions internes aux ATC, cela peut concerner, par exemple, le changement générationnel et le départ des membres fondateurs de l'ATC, l'arrivée de nouveaux membres, etc. Pour une entité productive, comme un ATC, les actions d'adaptation peuvent porter sur plusieurs volets: les activités de production (ajout ou suppression de produits, volume d'activité), les moyens de production (nouveaux investissements en bâtiment, augmentation ou baisse de la main-d'œuvre, réorganisation du travail, introduction de nouvelles technologies, etc.), les activités d'approvisionnement (fournisseurs, en particulier le renouvellement des adhérents pour les ATC) et/ou la commercialisation (nouveaux marchés, volumes de vente, etc.).

La dimension relative à la continuité renvoie à l'inertie de l'organisation face aux bouleversements. Cette inertie permet une stabilité propice à la survie de l'organisation dans un environnement changeant (Hannan et Freeman, 1989). C'est le principe de préserver l'essence de l'entité de l'organisation traduite par ses valeurs fondatrices, l'essentiel du projet initial par-delà les changements. Plusieurs éléments composent un facteur de stabilité et contribuent ainsi à constituer et/ou conforter ces valeurs fondatrices, comme l'histoire, les relations équitables et la justice sociale, la gestion privilégiant l'investissement à long terme (Mignon, 2009), la culture d'entreprise (Godelier, 2009), etc. Pour les exploitations agricoles, on peut citer le rôle de l'éthique (Macombe, 2009) ou de la coutume (de Bortoli et Palu, 2009) dans la pérennité de ces organisations. Pour les ATC, ces valeurs fondamentales concernent les valeurs de la coopération (Mondy, 2015): la solidarité entre les agriculteurs adhérents, la participation des adhérents à la vie de l'ATC, l'ancrage territorial de l'ATC. La dimension territoriale caractérise à la fois la réponse que peut apporter la structure collective aux enjeux territoriaux (Amblard et al., 2018) et l'approche intersectorielle de l'action collective avec la rencontre entre des acteurs agricoles et non agricoles (Auclair et al., 2015).

\subsection{Méthodologie}

11 Les ATC se sont structurés en coopératives d'utilisation de matériel agricole (Cuma) ou en organisations de producteurs. Les collectivités territoriales ont souvent accompagné la mise en place de ces formes à travers la procédure d'ateliers-relais et l'attribution d'aides publiques, dans le cadre de politiques d'aménagement et de développement rural. Ces soutiens se poursuivent aujourd'hui, notamment par des actions dans le domaine de l'alimentation (Naves, 2016), par la gestion foncière ou la diversification des exploitations agricoles (Guiomar, 2011).

Le Sud-Ouest est, en France, le territoire qui a connu le plus grand nombre de créations d'ATC (Mundler et Valorge, 2015). En effet, le recours à l'action collective pour la diversification y a été précoce et a bénéficié du soutien des fédérations locales des Cuma. Les ATC viennent ainsi combler un vide en matière de prestation de service, particulièrement pour les agriculteurs qui n'arrivent pas à bénéficier des services des structures individuelles de transformation. Ces dernières, si elles sont présentes sur le territoire, privilégient souvent les gros clients et/ou les plus anciens. Pour sélectionner leurs bénéficiaires, les structures individuelles pratiquent par exemple des tarifs élevés pour les clients occasionnels. De plus, ces structures individuelles ne couvrant pas l'essentiel des besoins, certains ATC ont élargi leur offre de service de prestation, au- 
delà de leurs adhérents. Pour comprendre les dynamiques qui ont présidé à la création des ATC, mais aussi celles qui permettent leur pérennité dans le temps, nous avons étudié $18 \mathrm{ATC}$ se situant dans la région d'Occitanie et quelques-uns dans les départements limitrophes. Le choix des ATC étudiés ne vise pas une représentation exhaustive des ATC du Sud-Ouest.

La collecte des données est basée sur la réalisation d'entretiens semi-directifs d'environ deux heures, avec des membres fondateurs et de nouveaux responsables des ATC. Le guide d'entretien aborde dans un premier temps les conditions de mise en place de ces structures collectives. Pour ce faire, nous avons mobilisé les quatre dimensions qui constituent un préalable à toute action collective, telles qu'abordées dans notre cadre analytique (cf. 1.1). Les questions portent donc sur les acteurs impliqués, les objectifs et les enjeux (motivations, projet initial, valeurs partagées, etc.), les règles de gouvernance (règlement intérieur, règles de bonnes pratiques, modalités de prise de décision) et les procédures de suivi et de gestion (contrôle du fonctionnement, suivi des résultats). La deuxième partie du guide d'entretien est consacrée à la question de la pérennité organisationnelle, avec ses deux dimensions : la capacité d'adaptation au changement et la continuité. La dimension changement des ATC est appréciée par les variables suivantes: (i) les évolutions des activités de production et de commercialisation (produits, prestations, formes de commercialisation, volumes), (ii) les évolutions des moyens de production (nouveaux investissements, recours au salariat, organisation du travail et projet de renouvellement de l'atelier) et (iii) le renouvellement des adhérents. La dimension continuité est étudiée à travers l'évolution des valeurs de l'ATC. Trois variables sont étudiées: (i) les valeurs de solidarité entre adhérents, (ii) les pratiques effectives de participation des adhérents, (iii) l'attention accordée à la revendication territoriale. Les données de l'enquête ont été exploitées, selon une démarche qualitative, de façon à faire apparaître une classification des ateliers fondée sur les deux éléments fondateurs de la pérennité des organisations: adaptation au changement et préservation des valeurs de coopération. Partant de cette classification, nous avons analysé les trajectoires des ATC de chaque groupe lors des dix dernières années. La représentation schématique des trajectoires est basée sur une appréciation qualitative du niveau de développement de ces ateliers (approché par le nombre d'adhérents et le volume d'activités), dans la période de temps ces dix dernières années (période commune à tous les ATC). L'appréciation de ce niveau de développement varie sur une échelle allant de faible à fort.

\section{Résultats}

\subsection{Les ATC dans le Sud-Ouest : projets d'action collective pour la diversification des exploitations}

\subsubsection{Une dynamique soutenue d'actions collectives}

Les ateliers étudiés sont pour la plupart situés dans la région Occitanie, ainsi que dans le département des Landes en région Nouvelle-Aquitaine (tableau 1). La majorité $(12 / 18)$ de ces ateliers ont un rayon d'action (localisation des adhérents) correspondant à l'échelle de quelques cantons, soit 30 à $50 \mathrm{~km}$. Le reste des ateliers couvrent une aire plus importante : trois ateliers couvrent la surface de leur département (rayon de 60 à $70 \mathrm{~km}$ ) et trois autres celle de plusieurs départements (rayon de 120 à $150 \mathrm{~km}$ ). 
Tableau 1. Caractéristiques des ATC

Les ateliers sont majoritairement consacrés à la transformation des viandes: de gros animaux (quatre ateliers), de volailles maigres ou grasses (cinq ateliers), ou des deux (cinq ateliers dits «multi-viandes»). Nous avons enquêté aussi deux ateliers de transformation de fruits, un atelier apicole et un atelier de production d'huile. Plusieurs ateliers de transformation de viandes envisagent ou commencent à intégrer des légumes pour enrichir leur gamme de plats cuisinés. Ces orientations productives reflètent la diversité du tissu agricole régional. Les statuts juridiques des ateliers sont majoritairement des coopératives (10 Cuma, 4 coopératives agricoles), trois ATC étaient en statut de Cuma à la création, sont devenus plus tard des Sarl (6T, 11M) ou SAS (16C); une ATC a choisi le statut de Scic pour pouvoir associer dans la même structure des collèges de producteurs, de collectivités territoriales et de consommateurs.

6 Neuf ateliers ont étendu leur champ d'activités, en plus de la transformation, à la commercialisation des produits. Cinq parmi eux disposent d'un magasin collectif. Huit ATC ont contribué à la création de marques collectives pour une meilleure valorisation des produits transformés. Ces marques s'appuient sur les spécificités des territoires des ATC, ce qui renforce par conséquent l'ancrage territorial de ces ATC. Notons aussi la contribution des ATC au développement territorial à travers, notamment, la création d'emplois directs (13/18 ont des salariés) et l'aide à l'installation des jeunes.

L'analyse de dates de création des ATC permet d'identifier trois vagues :

- les ATC fondés entre 1974 et 1999, qui constituent les premiers pas de la transformation agroalimentaire paysanne, en dehors du système agro-industriel; certains projets des premiers ateliers (par exemple $3 \mathrm{C}, 4 \mathrm{M}, 5 \mathrm{~V}$ ) ont mis longtemps à aboutir. Ils se heurtaient à différentes difficultés (réticences des organismes de développement agricole qui ne reconnaissaient pas cette forme de diversification agricole, difficultés financières, car ils étaient souvent portés par des producteurs manquant de moyens, projets portés par des néo-ruraux). Ce sont donc des histoires de projets alternatifs, de groupes soudés dans l'adversité, qui ont été à la base de la création de ces ATC ;

- les ATC créés entre 2000 et 2009 (12G, 13V, 14D) qui traduisent l'aboutissement de nouveaux projets assez semblables à leurs aînés. Ils sont créés plus facilement, la diversification est moins déconsidérée par la profession agricole, en raison de son importance économique ; le succès des pionniers permet de tenter de reproduire leur démarche. Dorénavant, les élus locaux soutiennent ce type de projets et peuvent même être à l'origine de leur création ;

- enfin les ATC créés depuis 2010 (15S, 16C, 17M, 18L) qui marquent le début d'une nouvelle vague de collectifs, influencés par le développement de la vente en circuits courts. Les responsables de ces ATC se considèrent comme porteurs de nouvelles formes d'activités agricoles qui satisfont les consommateurs, et mettent en avant leur volonté de faire exister un collectif fort autour de l'ATC.

\subsubsection{Processus de mise en place des ATC : les ressorts d'une action collective}

La mise en place de ces nouvelles structures de coopération est le résultat de dynamiques singulières, et qui dépassent souvent les frontières du secteur agricole. Le cadre analytique de l'action collective nous permet d'identifier dans les paragraphes suivants les caractéristiques partagées dans ces dynamiques. 

agriculteurs engagés dans la diversification à travers la transformation et la vente directe, des élus locaux qui expriment la volonté de créer des emplois ou de maintenir des exploitations agricoles et des agents de développement agricole (réseau Civam, FD Cuma, chambre d'agriculture, Mission agro-alimentaire Pyrénées, etc.) soucieux d'accompagner les agriculteurs dans leurs projets. Ces trois catégories de groupes participent à toutes les dynamiques de création d'ATC, mais le groupe leader diffère d'un cas à l'autre. Dans dix des dix-huit ATC (par ex. 1R, 3C, 5V, 9S, 13V), le projet de création est porté par les producteurs animés par l'idée de faire vivre de petites exploitations agricoles confrontées aux contraintes agricoles locales (par exemple pour 2D, exploitations situées en zone défavorisée, objet d'un processus de désertification avancé dans les années 1970, la densité de population est de 14 habitants au $\mathrm{km}^{2}$, avec une population vieillissante). Cinq autres ATC (2D, 4M, 6T, 8C, 11M) sont nés de dynamiques impulsées par un tandem de producteurs et d'élus locaux, traduisant une forte volonté de développement local par la diversification. Dans deux cas (16C, 18L), les élus locaux ont été à l'origine de la dynamique de création. Enfin, un ATC (atelier $12 \mathrm{G})$ est né d'une initiative portée par les élus de la chambre d'agriculture.

L'objectif principal est économique. En effet, ces ateliers représentent une solution de diversification pour développer, voire maintenir des exploitations. L'un des objectifs affichés par les fondateurs est de capter, par la transformation et la vente en circuits courts, une valeur ajoutée par rapport à la voie traditionnelle de commercialisation en filière longue. Un second objectif, affiché surtout par les responsables d'ATC de la première (entre 1974 et 1999) et de la troisième (à partir de 2010) vague, consiste à développer des projets alternatifs au modèle productiviste qui prône la spécialisation et l'intégration à la filière longue. Ne pouvant pas le faire à titre individuel par manque de moyens, ils recourent alors aux ATC pour le réaliser. Les ATC représentent pour eux un moyen d'autonomie par rapport aux opérateurs économiques des filières longues. Le troisième objectif est celui de la contribution au développement du territoire, à travers notamment la création des marques collectives, la contribution à l'emploi, le maintien des exploitations et l'aide à l'installation. Enfin, un quatrième objectif est d'ordre social (11 des 18 ATC) : l'atelier est vu comme un lieu de socialisation, d'entraide, de partage et de vie collective.

En matière de gouvernance, les fondateurs des ATC ont choisi des statuts coopératifs qui traduisent une volonté de s'ancrer dans les principes de la participation et de la mobilisation collective. Des règles sont établies pour l'adhésion, la répartition des responsabilités, le fonctionnement, l'utilisation de l'atelier, le respect des normes réglementaires, etc. Dans la majorité des ATC, des règlements intérieurs sont définis et arrêtés. Toutefois, dans la pratique, la participation effective des adhérents, le respect des règles restent variables. Plusieurs facteurs entrent en jeu, notamment le type de gestion des responsables de l'atelier ou le nombre d'adhérents. On constate par exemple que certains responsables d'ATC perpétuent une gouvernance qui repose sur un petit nombre de membres réellement actifs, se relayant pour les responsabilités au sein du bureau. Dans ce cas, la gouvernance est basée sur la confiance entre les membres. Dans les ateliers de création récente, on pratique une gouvernance qui peut s'inspirer de celle des ateliers de première génération : grande mobilisation collective, rappel régulier des objectifs, des règles en vigueur, mais cela est encadré par des règlements intérieurs contraignant à la participation aux activités décisionnelles 
(obligation de présence aux réunions, renouvellement anticipé des bureaux, etc.). Dans certains ATC, on exige de tous les adhérents une participation à un minimum de travail collectif (journées de travail mutualisé) pour maintenir l'esprit du collectif.

Enfin, pour les procédures de gestion et de suivi de résultats, il y a une réelle différence entre les ateliers. De manière générale, le suivi administratif et financier est assuré par le secrétariat de l'atelier, souvent un adhérent bénévole. Dans certains ateliers, c'est un salarié polyvalent (à temps partiel) mis à disposition dans l'atelier par une mairie (4M) ou la Fédération départementale des Cuma (9S) qui assure cette mission. Le secrétariat de l'atelier est aussi en charge de la facturation, des relations avec les coopérateurs, de l'administratif et de la gestion du planning d'utilisation. Les ateliers qui ont une personne qualifiée pour la gestion administrative et le suivi (3C, 17M) ont su éviter les répercussions des crises structurelles ou conjoncturelles (manque de renouvellement d'adhérents, crise de filière avicole, par exemple) grâce notamment à la vigilance des gestionnaires et leur capacité d'anticipation.

\subsection{Pérennité des ATC}

\subsubsection{Des structures plutôt pérennes à trajectoires multiples}

Les résultats montrent que la majorité des ATC est pérenne. Les deux tiers ont su s'adapter et garder les valeurs fondatrices (figure 1) alors que le restant a connu une évolution différente. Selon leurs trajectoires, nous distinguons quatre types. Les premier et deuxième types sont des ateliers pérennes, partagés entre une dynamique de maintien (huit ateliers) et une dynamique de croissance (quatre ateliers). Les troisième et quatrième types concernent les ateliers non pérennes avec deux trajectoires d'évolution, à savoir les ateliers ayant cessé leur activité (au nombre de trois) et ceux qui ont été repris par des particuliers (au nombre de trois).

Figure 1. Positionnement des ATC selon les variables de la pérennité

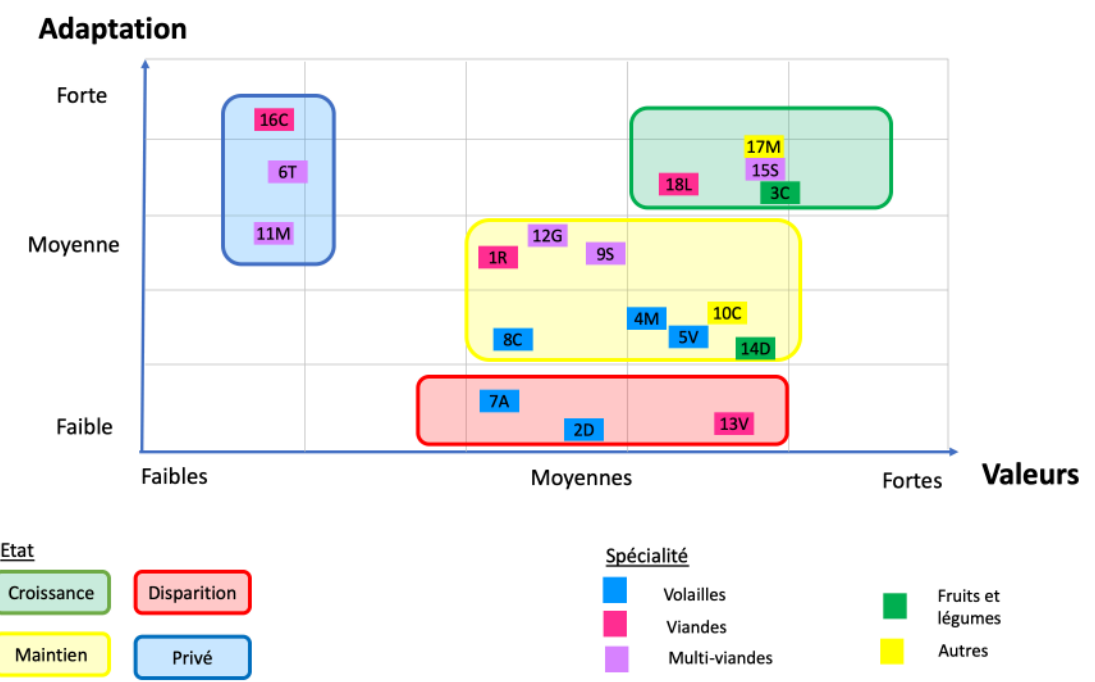

24 Le premier type, les ateliers en croissance, a été marqué par de gros changements pour s'adapter aux évolutions structurelles et conjoncturelles: réaliser de nouveaux investissements, diversifier ou élargir la gamme des activités et/ou renouveler le 
collectif d'adhérents. Par exemple, dans le contexte de crise avicole de 2015 et 2017, les responsables de l'atelier $15 \mathrm{~S}$ ont entrepris des investissements nécessaires pour rajouter une nouvelle chaîne de production de volailles maigres. Puis, dans l'optique de la labellisation en agriculture biologique (AB), ils ont abandonné les canards gras pour une diversification vers d'autres viandes en $A B$ (porcs, ovins, veaux). Enfin, dans un objectif de valorisation des bas morceaux, les responsables envisagent de créer une légumerie pour produire des plats cuisinés. Cet atelier connaît un fort taux de renouvellement d'adhérents avec une bonne implication de leur part. On peut citer un autre exemple, celui de l'atelier $3 \mathrm{C}$, où les responsables ont montré une grande capacité d'anticipation. Ils ont entrepris une diversification commerciale importante (création d'une marque collective, commercialisation d'une partie des produits transformés). Ils ont développé aussi de nouvelles activités, en plus de la transformation des fruits, comme la production de fruits secs et d'huile d'olive. De plus, dans ces deux exemples, les responsables des deux ateliers prêtent une attention particulière à l'entretien des valeurs du collectif. Ils réalisent des journées portes ouvertes avec une transformation collective. Ils veillent à inclure des agriculteurs en $A B$ et en conventionnel $(3 C)$ ou ils exigent la présence des nouveaux entrants aux réunions du conseil d'administration (15S).

La trajectoire type des ateliers en croissance (figure 2) montre que dans un premier temps, on cherche à "faire tourner » l'outil pour assurer des revenus dans la période de démarrage où les charges financières sont élevées. Puis dans un deuxième temps, ces ateliers cherchent à avoir des volumes assez importants et subsidiairement un groupe d'adhérents assez nombreux (3C et 18L). Ensuite, ces ateliers répondent à l'évolution de la demande: ils facilitent l'arrivée d'adhérents et investissent pour accueillir de nouvelles activités (projet de moulin à huile de l'atelier $3 \mathrm{C}$ ) ; ils prennent en compte les évolutions des marchés (prospection des marchés de restauration hors domicile) et innovent aussi dans les produits proposés (fabrication de steaks hachés).

Figure 2. Trajectoire type des ateliers en croissance

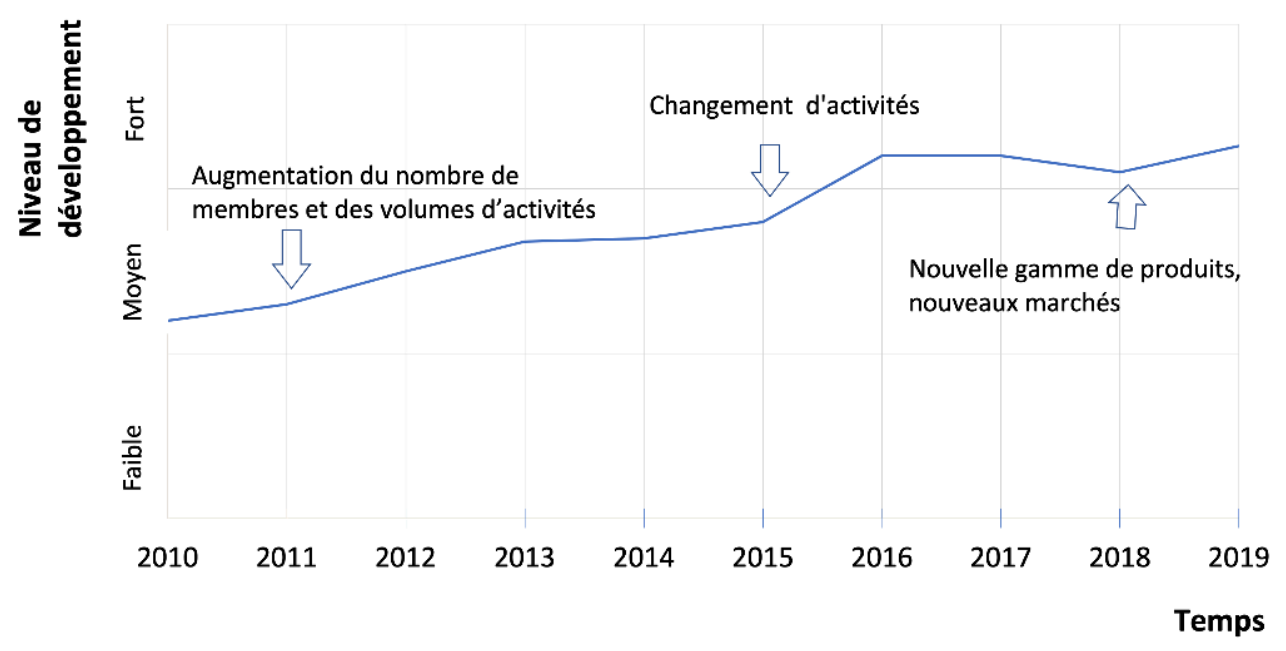

Le deuxième type, à savoir les ateliers en maintien, a pu connaître également de grands changements à la suite de crises. Par exemple, en réaction à la crise aviaire, les responsables de l'atelier $9 \mathrm{~S}$ ont introduit une nouvelle activité de transformation de porcs, qui correspond aux mêmes normes sanitaires que les volailles. L'atelier 14D a 
ajouté une ligne de production de châtaignes, à côté de la transformation des pommes, en réaction à la baisse d'activité. Dans les deux cas, l'atelier rencontre des difficultés économiques qui impactent la participation des adhérents et posent le problème du renouvellement des coopérateurs. De plus, les valeurs de coopération sont bousculées : manque de participation aux postes de responsabilité (14D) ou développement d'une partie des activités de transformation de volaille dans des fermes en parallèle de l'atelier. On a observé dans les ateliers $5 \mathrm{~V}$ et $9 \mathrm{~S}$ des dysfonctionnements dus au manque de respect des règles (fraude dans l'utilisation de l'atelier, non-paiement des factures dues à l'atelier) qui ont entaché la confiance et la solidarité entre les adhérents. Les nouveaux responsables ont établi de nouvelles règles, et ils ont adopté un style de gestion plus rigoureux. D'autres ATC (1R et 4M) ont développé de nouveaux projets permettant d'installer des jeunes : rajouter une ligne de steak haché, de légumineuses, travailler sur l'autonomie énergétique des ateliers, etc.

La trajectoire type des ateliers en maintien (figure 3) montre qu'après une période de croissance et d'augmentation des membres et des volumes et/ou des débouchés apparaissent divers problèmes. Les plus graves sont liés à la gestion des ressources humaines : un manque de renouvellement des adhérents qui entraîne une baisse des volumes et la perte de rentabilité, un départ de salariés essentiels au fonctionnement. Mais ces ateliers parviennent à prendre des décisions importantes pour maintenir la dynamique collective : diversification des activités (9S, 14D), une modification cruciale d'orientation (1R, 4M), et/ou nouvelles règles de fonctionnement $(5 \mathrm{~V}, 9 \mathrm{~S})$.

Figure 3. Trajectoire type des ateliers en maintien

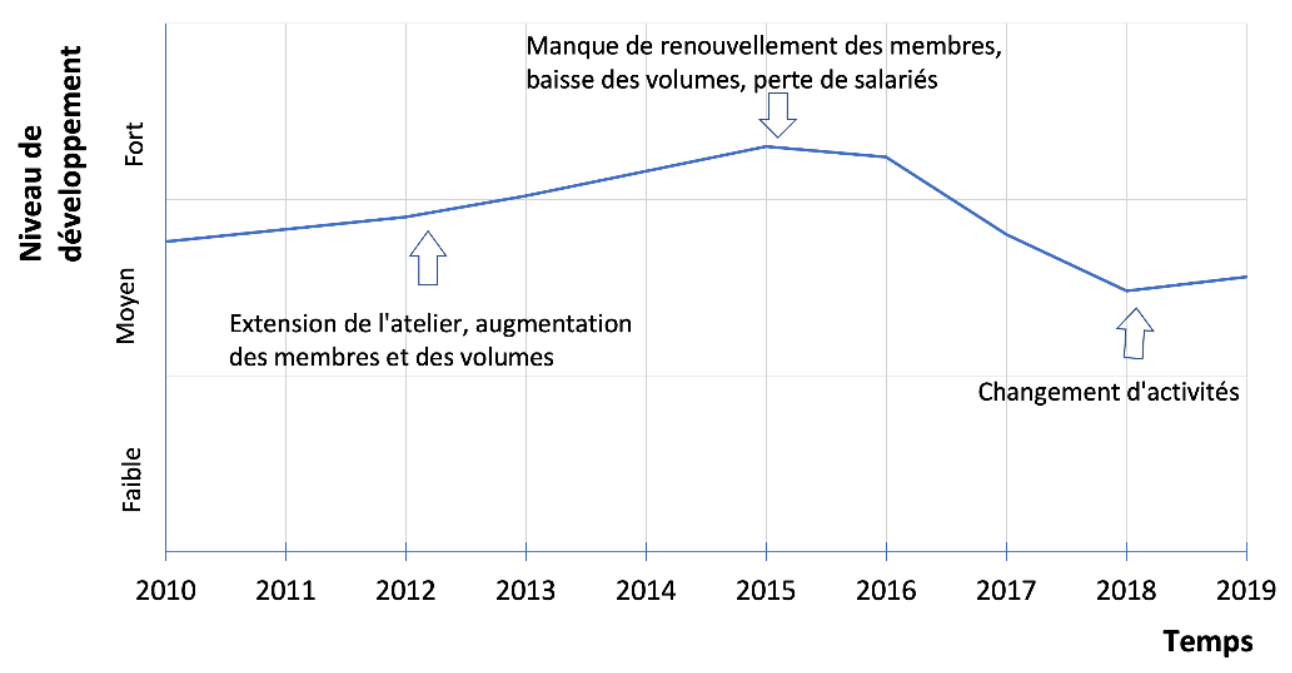

Le troisième type, les ateliers ayant disparu, est caractérisé par une incapacité à réagir à des événements comme les modifications récurrentes des normes sanitaires, ou les épisodes de grippe aviaire. Ces ateliers présentent pourtant un attachement aux valeurs coopératives. Par exemple, l'atelier $13 \mathrm{~V}$ a dû fermer ses portes à cause de la fermeture de l'abattoir local situé à $15 \mathrm{~km}$. Mais il était déjà en difficulté économique et n'atteignait que la moitié du niveau d'activité suffisant pour faire face aux coûts d'amortissement et de fonctionnement. Les ateliers $2 \mathrm{D}$ et 7A se sont trouvés dans le même cas. La crise aviaire cumulée avec le besoin de gros investissements pour la mise aux normes et le manque de renouvellement des adhérents ont conduit à leur fermeture. 
La trajectoire type de ces ateliers (figure 4) montre une baisse progressive des effectifs et des volumes transformés, entraînant des difficultés financières. Le vieillissement des adhérents a souvent provoqué une démobilisation: les décisions d'adaptation ont été repoussées ou refusées par des adhérents en fin de carrière (2D, 7A).

Figure 4. Trajectoire type des ateliers en déclin ou disparus

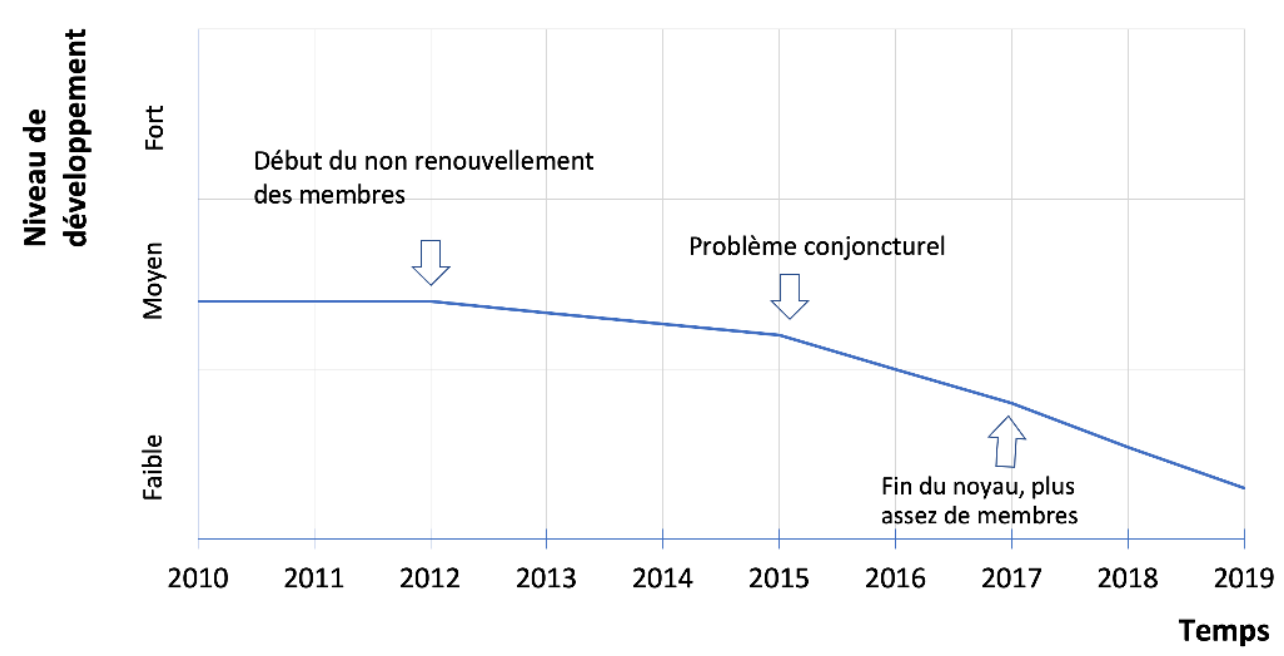

Enfin, le quatrième type d'ateliers, ceux devenus des structures individuelles, a été marqué par le manque d'engagement des adhérents cumulé à des difficultés de gestion. D'anciens salariés les ont rachetés (chef d'atelier pour $6 \mathrm{~T}$, bouchers pour 11M et $16 \mathrm{C}$ ) ; le rôle grandissant de certains salariés affectés à des postes clés peut contribuer à transformer ces structures en entreprises commerciales individuelles. Ces salariés devenus repreneurs maîtrisent des compétences techniques indispensables (les bouchers dans les ateliers $11 \mathrm{M}$ et $16 \mathrm{C}$ ), ou sont seuls à connaître l'ensemble des dossiers administratifs et comptables (6T).

\subsubsection{Les facteurs de pérennité des ATC}

31 Nous avons recensé trois facteurs qui agissent fortement sur la pérennité des ateliers : les acteurs, la capacité d'anticipation, le rôle de l'atelier dans le développement territorial.

32 Au premier rang des facteurs de pérennité, on trouve la capacité à renouveler les membres qui animent et utilisent l'atelier. Les ateliers en croissance ou en maintien ont accueilli de nouveaux membres, avec une ouverture sur un nouveau public. Nous avons enquêté des ateliers qui comptent parmi leurs adhérents des agriculteurs en $A B$ et en conventionnel (ce qui les conduit à une organisation rigoureuse des phases de transformation), des acteurs issus du territoire ou allochtones. Ces groupes composites considèrent leur hétérogénéité comme une richesse. Dans les ateliers en croissance, l'accent est mis sur la nécessité de former les nouveaux entrants au fonctionnement de l'atelier, aux moyens techniques comme à la gestion, dans le but de les préparer à assumer un jour des responsabilités.

Dans le cas opposé, le manque de renouvellement dans la prise de responsabilités, une forte dépendance aux compétences de salariés clés ou le non-respect des règles constituent des causes de fragilité des ateliers qui ont montré une faible capacité 
d'adaptation (1R, 8C, 12G), de ceux qui ont arrêté leur activité (2D, 7A, 13V) ou qui sont devenus des structures individuelles $(6 \mathrm{~T}, 11 \mathrm{M}, 16 \mathrm{C})$. Nous avons vu dans ces ateliers des effectifs qui se sont délités. Devant ces difficultés, les dirigeants font état d'une forme de lassitude, voire d'épuisement, mais résistent pour sauver un outil essentiel à leurs yeux. Certains ateliers sont confrontés à de forts déséquilibres entre apporteurs qui conduisent à des formes de privatisation larvée. Il peut arriver par exemple qu'un adhérent devienne le plus gros utilisateur de l'outil, lui permettant ensuite de devenir une force économique au sein de la structure, voire son président ou l'hébergeur de l'activité. Il est compréhensible que, dans ce cas de figure, faire la part entre intérêts particuliers et collectifs devient une affaire ardue.

Le deuxième facteur de pérennité est la capacité d'anticipation des changements. Celle-ci facilite l'adaptation de l'atelier aux évolutions lentes. En anticipant l'évolution des adhésions, les responsables d'ateliers élaborent de nouveaux produits et organisent la communication autour des produits et de l'outil: l'atelier $15 \mathrm{~S}$ a radicalement changé ses productions, tandis que les responsables des ateliers $3 \mathrm{C}$ et $14 \mathrm{D}$ ont entrepris de grands changements, évoqués plus haut: développement d'une marque collective de l'atelier, introduction de nouvelles activités.

L'absence de capacité d'anticipation peut conduire à remettre en cause la pérennité des ateliers dans des contextes de difficultés conjoncturelles, à l'instar de la filière du canard gras qui a connu une transformation profonde. En effet, depuis 20 ans, production et consommation ont énormément augmenté, les GMS captant la majeure partie de ce progrès; or les adhérents des ateliers étudiés revendiquent un modèle différent : quantités limitées, distribution exclusive... qui limite leur expansion. Plus récemment, une partie des consommateurs les plus jeunes se détournent des produits issus du gavage au nom du bien-être animal, les agriculteurs sont à l'heure actuelle dans l'incapacité d'élaborer une réponse à ces attentes. Enfin, dans les fermes de petite taille, les règlements pris après le second épisode de grippe aviaire en 2017, qui interdisent de mélanger les espèces et contraignent à une reconfiguration des parcs d'élevage et des circulations, la solution la plus aisée est d'abandonner les volailles grasses. Les ateliers qui ont conçu (parfois exclusivement) des lignes de transformation spécifiques aux palmipèdes gras ont été très affectés et n'ont pas tous eu la capacité de réagir (deux des trois ateliers fermés, trois des ateliers en maintien les plus fragiles entrent dans cette catégorie).

Le troisième facteur est celui du rôle des ateliers dans le développement territorial. En effet, au-delà d'un apport indéniable au développement agricole de proximité, les ateliers les plus dynamiques ont été réfléchis en étroite connexion avec le territoire dans lequel ils évoluent. Les agriculteurs, les élus et la profession agricole ( $3 C$ et $12 \mathrm{G}$ ) à l'origine de la création de certains ateliers les ont considérés comme des outils nécessaires au maintien d'une activité dans un espace parfois élargi au-delà des limites départementales (l'atelier $3 \mathrm{C}$ compte 345 membres issus de 5 départements). Le rôle d'appui de l'outil de transformation à l'installation agricole ou au maintien est largement reconnu; les emplois de l'atelier, occupés par des habitants du territoire proche du site, comptent autant que ceux des fermes qui le fournissent. Vu cette importance, certains ateliers étaient fortement soutenus par les élus locaux (certains ateliers ont été créés par des élus, d'autres ont reçu leur appui financier ou la caution de la collectivité territoriale). Toutefois, cette forte implication des élus peut s'avérer une source de fragilité dans l'analyse de la pérennité. En effet, dans le cas où le soutien 
des élus est fort, plus encore lorsque l'atelier est leur création, une défaite électorale peut avoir des conséquences extrêmement dommageables (perte de soutien financier, renégociation de loyers...) et peut précariser fortement l'outil comme les fermes qui en dépendent.

\section{Discussion}

37 Ces résultats éclairent différentes dimensions du processus de création d'actions collectives d'agriculteurs et leurs conditions de pérennité dans le temps. Nous discuterons dans un premier temps des éléments saillants du processus de mise en place des ATC, en partant du cadre d'analyse de l'action collective. Puis nous analyserons les conditions de pérennité de ces formes de coopération de proximité.

Les processus de création des ATC montrent que les principaux acteurs de l'action collective sont les producteurs eux-mêmes, mais aussi des acteurs non agricoles (des élus proches du monde agricole ou sensibles au développement local) et des acteurs de développement agricole ouverts à ces projets (les réseaux des Cuma et des Civam). L'engagement des acteurs non agricoles dans certains ATC est à souligner. S'il était peu commun dans le contexte des années 1980 (la question du développement agricole était exclusivement du ressort de la politique sectorielle du ministère de l'Agriculture et soumise au système de cogestion État-Syndicats majoritaires), il l'est moins aujourd'hui (Naves, 2016). Différents travaux ont montré l'implication croissante des conseils régionaux dans le développement des formes d'agriculture territorialisées (Trouvé, 2009 ; Blanc, 2012) ou celles d'autres acteurs non agricoles (intercommunalités, pays, parcs naturels, etc.) dans les Projets alimentaires territoriaux (Maréchal et al., 2018). On peut considérer à ce propos que certains ATC étaient précurseurs de cette approche systémique et territoriale du développement. Des travaux à l'échelle européenne mettent en avant la pertinence de cette approche intégrative et la considèrent comme un changement de paradigme de développement rural (Van der Ploeg et al., 2000; Renting et al., 2003).

En termes d'objectifs, les agriculteurs s'appuient sur les ATC pour développer des activités de diversification génératrices d'une valeur ajoutée importante, comparée à la commercialisation en filière longue (Gafsi et Delevers, 2015). Ils s'inscrivent, pour plusieurs d'entre eux, dans des systèmes agro-alimentaires alternatifs (Goodmann, 2004; Maye, 2013). C'est ce que Van der Ploeg (2014) qualifie de logique d'autonomisation par rapport au modèle agro-industriel. Cet objectif de l'action collective comme levier de recherche d'autonomie est aussi mis en avant par les travaux de Lucas et Gasselin (2018) à propos des expériences d'actions collectives d'éleveurs dans le cadre des Cuma pour le développement de pratiques agroécologiques et une valorisation optimale des ressources. Cette perspective d'autonomie est également un trait marquant des collectifs d'agricultures «alternatives » étudiés par Demeulenaere et Goulet (2012).

En ce qui concerne la pérennité, nous constatons que la majorité des structures étudiées sont pérennes. Il y a eu préservation des valeurs fondatrices du projet initial et changements multiples pour saisir des opportunités de développement ou pour surmonter des difficultés conjoncturelles. Ce résultat contraste avec l'évolution de la majorité des coopératives agricoles traditionnelles qui ont connu une mutation structurelle profonde ces 40 dernières années (Mauget, 2008): concentration et 
constitution de groupes coopératifs complexes en développant des filiales de statuts non coopératifs et des alliances avec des entreprises capitalistes. Mais si on se réfère aux Cuma (dix des dix-huit ATC sont des Cuma), ces résultats sont en cohérence avec la trajectoire de ces structures de coopération de proximité (FNCUMA, 2017) : le nombre des Cuma est stable autour de 12000 , il y a donc maintien d'une dynamique collective avec un chiffre d'affaires en augmentation et une recomposition des fonctions de la Cuma pour s'adapter à la conjoncture.

41 Cette pérennité des ATC est le fruit de la capacité à maintenir la mobilisation des acteurs autour du projet et le renouvellement des membres. Dans ces ATC, l'action collective reste un facteur cardinal de l'identité ; certains ateliers maintiennent des pratiques collectives de travail et de prise de décision. D'autres accordent une attention particulière à l'accueil de nouveaux membres, en élargissant le champ des activités de l'atelier et en déployant des actions d'intégration de ces nouveaux membres. Ce facteur de renouvellement des membres et de partage de valeurs coopératives joue conjointement avec une logique de gestion proactive qui anticipe les changements et saisit les opportunités de développement pour lancer de nouveaux projets.

Nous avons vu, par contre, que pour certains ateliers, le manque d'adhésion des membres ou l'absence de renouvellement des adhérents conduisent à des difficultés amenant à menacer leur pérennité. Dans ce contexte, le renouvellement de la génération des fondateurs n'est pas assuré ; des dirigeants lassés d'une mission exercée trop longtemps finissent par abandonner. Mais ce facteur de manque de relève générationnelle se manifeste, pour les cas étudiés, conjointement avec le manque de capacité d'anticipation et le cumul des difficultés économiques des ATC concernés. L'identification de ces facteurs de risque (relève générationnelle et/ou difficultés conjoncturelles) invitent les structures d'accompagnement (FD Cuma, Civam, chambres d'agriculture, etc.) à porter une attention particulière à ces facteurs et à travailler sur les conditions permettant de les surmonter.

Au-delà de ces facteurs internes, d'autres facteurs externes comme les évolutions du marché des circuits courts ou l'évolution des normes sanitaires (à la suite des crises aviaires par exemple) peuvent impacter la pérennité des ATC. Également, les contextes territoriaux jouent dans le processus de mise en place des ateliers collectifs comme dans leur pérennité. Ceci est mis en évidence par le rôle des élus dans la mise en place des ATC, et par conséquent l'impact de l'évolution de leur rôle à la suite des changements de majorité après des élections. Ce défi se pose pour plusieurs initiatives agro-alimentaires impliquant des collectivités territoriales (Blanc, 2012). La dimension territoriale est inhérente à toute action collective de proximité, nos résultats rejoignent les récentes études sur les conditions territoriales de développement de l'action collective (Amblard et al., 2018 ; Corade et al., 2019).

\section{Conclusion}

44 L'expérience des ATC montre des dynamiques singulières d'action collective de proximité, initiées par des collectifs de producteurs et soutenues par des collectivités territoriales et des réseaux d'accompagnement de développement agricole. Animés par des objectifs de création de valeur ajoutée par le biais de la transformation des produits agricoles et par la contribution au développement territorial, ces collectifs de producteurs ont pu mettre en place, non sans difficultés surtout dans la première vague 
(années 1980 et 1990), des projets innovants de coopération agricole. Forts de cette expérience, de la reconnaissance de l'importance de la diversification agricole et de leur rôle dans le développement territorial (création, et maintien des emplois, création de marques collectives valorisant les spécificités des territoires...), les ATC de la deuxième et troisième vague (années 2000 et 2010) sont confrontés à de nouveaux défis relevant de leur capacité d'innovation et de réponse aux changements conjoncturels. La question de la pérennité de ces organisations de coopération est ainsi une préoccupation importante pour les leaders de ces collectifs.

L'étude des trajectoires des ATC révèle que la majorité de ces collectifs sont pérennes, témoignant d'une grande capacité d'adaptation aux changements structurels et conjoncturels, et d'une attention particulière à préserver les valeurs de coopération. Plusieurs ATC ont pu surmonter d'importantes difficultés structurelles et/ou conjoncturelles. Ceci conduit à réfléchir, en matière de suites éventuelles à la présente étude, aux facteurs de résilience des formes d'action collective de production. On peut faire l'hypothèse de l'importance de certains facteurs comme ceux de la proximité (Torre, 2014 ; Polge et al., 2016), de la figure de réseau comme mode d'action collective (Demeulenaere et Goulet, 2012), de l'agilité et la souplesse de ces formes d'action collective (Charbonnier-Voirin, 2011), etc.

Toutefois, certains ATC ont connu des trajectoires de déclin qui ont abouti soit à leur disparition, soit à leur transformation en structures individuelles. Ce manque de pérennité met en avant, entre autres facteurs, les difficultés de certains ATC à renouveler la génération des fondateurs et/ou à s'adapter aux changements structurels et conjoncturels. Cette question de relève générationnelle peut faire l'objet d'un travail approfondi, en prolongement à la présente étude, et en menant une analyse comparative avec les Cuma. En effet les Cuma représentent la forme de coopération de production la plus proche des ATC, et la question de la relève générationnelle ne semblerait pas poser de difficultés pour eux.

\section{BIBLIOGRAPHIE}

Amblard L., Berthomé G. E. K., Houdart M., Lardon S., 2018, « L'action collective dans les territoires. Questions structurantes et fronts de recherche ", Géographie, économie, société, vol. 20, $\mathrm{n}^{\circ} 2$, p. 227-246.

Auclair F., Mondy B., Terrieux A., 2015, « Ancrage et impact territorial des ATC : histoire d'une rencontre ", in Mundler, P., Valorge, F. (eds.), Ateliers de transformation collectifs. Enjeux et outils pour réussir, Dijon, Educagri, p. 219-234.

Barraud-Didier V., Henninger M.-C., Anzalone G., 2012, « La distanciation de la relation adhérentcoopérative en France ", Études rurales, n 190, p. 119-130, https://doi.org/10.4000/etudesrurales. 9700. 
Blanc J., 2012, « Construire l'alternative agro-alimentaire : ressorts sociaux et politiques du déploiement des AMAP en Île-de-France », Norois, n²24, p. 21-34, https://doi.org/10.4000/ norois.4256.

Charbonnier-Voirin A., 2011, « Développement et test partiel des propriétés psychométriques d'une échelle de mesure de l'agilité organisationnelle ", M@n@gement, vol. 14, n² 2, p. 119-156, https://doi.org/10.3917/mana.142.0119.

Corade N., Lemarié- Boutry M., Gomez A., Pérès S., 2019, « Existe-t-il une ambiance territoriale favorable au développement et au maintien des circuits courts et de proximité ? ", Communication aux JRSS de la SFER, 12-13 décembre 2019, Bordeaux.

Crozier M., Friedberg E., 1977, L'acteur et le système, Paris, Seuil.

De Bortoli D., Palu P., 2009, « Le système maison comme déterminant de la pérennité organisationnelle », Revue française de gestion, vol. 2, n 192, p. 141-150, https://www.cairn.info/ revue-francaise-de-gestion-2009-2-page-141.htm.

Demeulenaere É., Goulet F., 2012, « Du singulier au collectif », Terrains \& travaux, vol. 1, n 20, p. 121-138, https://doi.org/10.3917/tt.020.0121.

Filippi M., Frey O., Mauget R., 2008, « Les coopératives agricoles face à l'internationalisation et à la mondialisation des marchés », Revue internationale de l'économie sociale, n 310, p. 31-51, https:// doi.org/10.7202/1021102ar.

FNCUMA, 2017, « Chiffres clés, édition 2017 », rapport FNCUMA.

Friedberg E., 1993, Le pouvoir et la règle. Dynamiques de l'action organisée, Paris, Seuil.

Gafsi M., 2017, « Les stratégies de diversification des exploitations agricoles. Enseignements théoriques et empiriques ", Économie rurale, n 360, p. 43-63, https://doi.org/10.4000/ economierurale.5257.

Gafsi M., Delevers L., 2015, « Impact des ateliers collectifs de transformation sur les performances socio-économiques des exploitations agricoles ", in Mundler, P., Valorge, F., (éds.) Ateliers de transformation collectifs Transformer collectivement ses produits agricoles dans les territoires, Dijon, Educagri Éditions, p. 235-258.

Girin J., 1990, « L'analyse empirique des situations de gestion : élément de théorie et de méthode », in A. C. Martinet (coord.), Épistémologie et sciences de gestion, Paris, Economica, p. 141-182.

Godelier É., 2009, « La culture d'entreprise », Revue française de gestion, vol. 2, n 192, p. 95-111, https://www.cairn.info/revue-francaise-de-gestion-2009-2-page-95.htm.

Goodman D., 2004, « Rural Europe redux ? Reflections on alternative agro-food networks and paradigm change $"$, Sociologia ruralis, vol. 44, $\mathrm{n}^{\circ} 21, \mathrm{p} .3-16$.

Guiomar X., 2011, « Les collectivités locales à la recherche d'une agriculture de proximité », Pour, vol. 2-3, n 209-210, p. 169-183, https://doi.org/10.3917/pour.209.0169.

Hannan M. T., Freeman J., 1989, Organizational ecology, Harvard university press, 384 p.

Hatchuel A., 2000, «Quel horizon pour les sciences de gestion? Vers une théorie de l'action collective ", in David A., Hatchuel A., Laufer R. (eds.), Les nouvelles fondations des sciences de gestion, Paris, Vuibert, p. 25-45.

Hatchuel A., Weil B., 1992, L'expert et le système, Paris, Economica, 264 p. 
Koulytchizky S., Mauget R., 2003, « Le développement des groupes coopératifs agricoles depuis un demi-siècle : à la recherche d'un nouveau paradigme ", Revue internationale de l'économie sociale $\mathrm{n}^{\circ} 287$, p. 14-40, https://doi.org/10.7202/1022208ar.

Lucas V., Gasselin P., Thomas F., Vaquié P.-F., 2014, « Coopération agricole de production : quand l'activité agricole se distribue entre exploitation et action collective de proximité », in Gasselin P., Choisis J.-P., Petit S., Purseigle F., Zasser S. (éd.), L'agriculture en famille : travailler, réinventer, transmettre, Paris, EDP Sciences, p. 201-222.

Lucas V., Gasselin P., 2018, « Gagner en autonomie grâce à la Cuma. Expériences d'éleveurs laitiers français à l'ère de la dérégulation et de l'agroécologie ", Économie rurale, n 364, p. 73-89, https://doi.org/10.4000/economierurale.5554.

Macombe C., 2009, «Éthique et proactivité agricole pour durer », Revue française de gestion, vol. 2, $\mathrm{n}^{\circ}$ 192, p. 127-140, https://www.cairn.info/article.php?

ID_ARTICLE $=$ RFG_192_0127\&DocId =146809.

March J. G., 1991, « Exploration and exploitation in organizational learning », Organization science, vol. $2, \mathrm{n}^{\circ} 1$, p. 71-87.

Maréchal G., Noel J., Wallet F., 2018, « Les projets alimentaires territoriaux (PAT) : entre rupture, transition et immobilisme? », Pour, vol. 2-3, n 234-235 p. 261-270.

Mauget R., 2008, « Les coopératives agricoles : un atout pour la pérennité de l'agriculture dans la mondialisation », Revue internationale de l'économie sociale, n 307, p. 46-57, https://doi.org/ 10.7202/1021194ar.

Maye D., 2013, « Moving Alternative Food Networks beyond the Niche », International Journal of Sociology of Agriculture and Food, vol. 20, n 3, p. 383-389.

Meinzen-Dick R., DiGregorio M., McCarthy N., 2004, « Methods for studying collective action in rural development », Agricultural Systems, vol. 82, p. 197-214.

Mignon S., 2009, «La pérennité organisationnelle : un cadre d'analyse », Revue française de gestion, vol. 35, n 192, p. 73-89, https://www.cairn.info/revue-francaise-de-gestion-2009-2page-73.htm ?contenu =resume.

Mondy B. (dir), 2010, « Vers une agriculture territorialisée : analyse des formes d'organisation collective et innovante de diversification agricole ", rapport d'étude pour le conseil régional de MidiPyrénées.

Mondy B., Terrieux A., 2010, « Vers une agriculture territorialisée, où s'alimentent les circuits courts ? - le rôle des ateliers collectifs de transformation agroalimentaire », in Traversac J.-B. (ed.), Circuits courts : contribution au développement régional, Dijon, Educagri, p. 89-101.

Mondy B., 2015, « Des premiers CUMA de diversification aux ATC, vers une évolution des formes coopératives de production ", in Mundler, P., Valorge, F. (eds.), Ateliers de transformation collectifs. Enjeux et outils pour réussir, Dijon, Educagri, p. 23-37.

Muller P., Gerbaux F., Faure A., Gerbaux F., 1989, Les entrepreneurs ruraux : agriculteurs, artisans, commerçants, élus locaux, Paris, L'Harmattan, 189 p.

Mundler P., Valorge F., 2015, Ateliers de transformation collectifs. Enjeux et outils pour réussir, Dijon, Educagri, 297 p.

Naves P., 2016, «L'encadrement des circuits courts. Du secteur agricole aux territoires ?», Économie rurale, $\mathrm{n}^{\circ} 355$, p. 3-19, https://doi.org/10.4000/economierurale.4978. 
Nicolas P., 1988, «Émergence, développement et rôle des coopératives agricoles en France. Aperçus sur une histoire séculaire ", Économie rurale, n 184-185-186, p. 116-122, https:// www.persee.fr/doc/ecoru_0013-0559_1988_num_184_1_3900.

Olson M. L., 1965, The Logic of Collective Action, Cambridge, MA, Harvard University Press.

Ostrom E., 1990, Governing the commons : The evolution of institutions for collective action, Cambridge, Cambridge university press.

Poisson M., Saleilles S., 2012, "À chaque collectif de producteurs sa recette », in Prigent-Simonin, A.-H. (ed.), Au plus près de l'assiette : pérenniser les circuits courts alimentaires, Dijon, Educagri Éditions, p. 117-137.

Polge É., Torre A., Piraux M., 2016, « Dynamiques de proximités dans la construction de réseaux socio-économiques territoriaux en Amazonie brésilienne », Géographie, économie, société, vol. 18, $\mathrm{n}^{\circ} 4$, p. 493-524.

Renting H., Marsden T. K., Banks J., 2003, « Understanding alternative food networks : exploring the role of short food supply chains in rural development », Environment and planning, vol. 35, $\mathrm{n}^{\circ}$ 3, p. 393-411.

Torre A., 2014, « Relations de proximité et comportements d'innovation des entreprises des clusters ", Revue française de gestion, vol. 5, n²42, p. 49-80, https://www.cairn.info/revuefrancaise-de-gestion-2014-5-page-49.htm.

Trouvé A., 2009, « Les régions, porteuses de nouveaux compromis pour l'agriculture ? ", Revue de la régulation, $\mathrm{n}^{\circ}$ 5, p. 1-22, https://doi.or/10.4000/regulation.7550.

Van der Ploeg J. D., Renting H., Brunori G., Knickel K., Mannion J., Marsden T. K., de Roest K., Sevilla-Guzman E., Ventura F., 2000, « Rural development : from practices and policies towards theory ", Sociologia Ruralis vol. 40, $\mathrm{n}^{\circ}$ 4, p. 391-408.

Van der Ploeg J.-D., Laurent C., Blondeau F., Bonnafous P., 2009, « Farm diversity, classification schemes and multifunctionality ", Journal of environmental management, vol. 90, Supplement $n^{\circ} 2$, p. 124-131.

Van der Ploeg J.-D., 2014, Les paysans du XXI ${ }^{e}$ siècle. Mouvements de repaysannisation dans l'Europe d'aujourd'hui, Paris, Charles Léopold Mayer Éditions, 217 p.

Vincq J.-L., Mondy B., Fontorbes J.-P., 2010, « La construction de la qualité fiable dans les réseaux alimentaires de proximité », Économie rurale, $\mathrm{n}^{\circ}$ 318-319, p. 5-19, https://doi.org/10.4000/ economierurale.2851.

\section{RÉSUMÉS}

Les ateliers de transformation collectifs (ATC) ont émergé au cours des 30 dernières années pour aider les agriculteurs dans leurs stratégies de diversification, en mutualisant différentes ressources productives. Si les travaux sur la coopération agricole ont été nombreux, les recherches portées spécifiquement sur les nouvelles formes de structuration collective restent cependant rares. L'objectif de cet article est d'apporter un éclairage sur les processus de création de ces structures collectives et de questionner leur pérennité. Nous mobilisons pour notre cadre d'analyse la théorie de l'action collective et le concept de pérennité. Sur le plan méthodologique, nous avons adopté une approche qualitative pour conduire une analyse rétrospective portant sur 18 ATC dans le sud-ouest de la France. Les résultats montrent que la pérennité des ATC dépend de leur processus d'adaptation aux changements structurels et conjoncturels ainsi que de leur 
aptitude à préserver des valeurs de coopération. Plusieurs trajectoires ont été observées : celles de croissance, de maintien, de déclin d'ATC ou sa transformation en structure individuelle. Les principaux facteurs de pérennité ont été mis en évidence, comme le renouvellement des membres, la capacité d'anticipation et le rôle de l'atelier dans le développement territorial. La contribution de cette recherche porte sur la capacité d'adaptation des structures d'action collective de producteurs, et sur la nécessité d'accorder une attention particulière aux facteurs de leur pérennité.

Collective transformation workshops (ATC) have emerged over the last thirty years to pool resources for farmers to diversify. Studies about agricultural cooperation are numerous, but those related to these new structures are relatively few. The purpose of this article is to shed light on the creation process of these collective structures and their sustainability over the time. Our analysis framework uses the theory of collective action and the concept of perenniality. We chose a qualitative methodological approach in order to carry out a retrospective analysis of 18 ATC in south-western France. The results show that, overall, there is a long-term perenniality of ATCs with a various adaptation process to structural and economic circumstances and a close attention to retain the values of cooperation. However, several trajectories have been observed, among which those of decline (disappearance) or its mutation into a individual structure. Several factors of perenniality were highlighted, such as the membership renewal, ability to anticipate, the role of the ATC role in territorial development. The contribution of this research concerns the adaptive capacity of collective action structures of producers, and the need to pay particular attention to the factors of their sustainability.

\section{INDEX}

Mots-clés : action collective, transformation agricole, diversification, trajectoire, pérennité Keywords : collective action, food processing, diversification, trajectory, perenniality

\section{AUTEURS}

\section{AGNÈS TERRIEUX}

Agnès Terrieux est maîtresse de conférences à l'ENSFEA et chercheuse au LISST-Dynamiques rurales (UMR LISST, 5193, CNRS-UT2J-EHESS-ENSFEA). Ses thématiques de recherche concernent la reterritorialisation de l'agriculture, les systèmes agroalimentaires territorialisés et les formes de diversification agricole.

agnes.terrieux@ensfea.fr

\section{MOHAMED GAFS}

Mohamed Gafsi est professeur à l'ENSFEA et directeur du LISST-Dynamiques Rurales (UMR LISST, 5193, CNRS-UT2J-EHESS-ENSFEA). Ses thématiques de recherche concernent la gestion durable des exploitations agricoles, les dynamiques d'innovation et d'action collective pour une transition agroécologique des exploitations agricoles et des systèmes agroalimentaires. mohamed.gafsi@ensfea.fr

\section{UGO FIASCHI}

Ugo Fiaschi est titulaire du master Gestion des territoires et développement local - Territoires ruraux. Ses travaux portent sur les actions collectives en appui à la diversification des 
exploitations agricoles.

hf_31@hotmail.fr 